\title{
Quasi-Conformal Flat Representation of Triangulated Surfaces for Computerized Tomography
}

\author{
Eli Appleboim, Emil Saucan, and Yehoshua Y. Zeevi \\ Electrical Engineering Department, Technion, Haifa, Israel \\ (eliap, semil,zeevi@ee.technion.ac.il)
}

\begin{abstract}
In this paper we present a simple method for flattening of triangulated surfaces for mapping and imaging. The method is based on classical results of F. Gehring and Y. Väisälä regarding the existence of quasi-conformal and quasi-isometric mappings between Riemannian manifolds. A random starting triangle version of the algorithm is presented. A curvature based version is also applicable. In addition the algorithm enables the user to compute the maximal distortion and dilatation errors. Moreover, the algorithm makes no use to derivatives, hence it is robust and suitable for analysis of noisy data. The algorithm is tested on data obtained from real CT images of the human brain cortex and colon, as well as on a synthetic model of the human skull.
\end{abstract}

\section{Introduction}

In many medical applications of image processing, such as medical imaging for noninvasive diagnosis and image guided surgery, a paramount importance lies in the process of two-dimensional representation by flattening of three-dimensional object scans. For example, it is often advantageous to present three-dimensional MRI or CT scans of the cortex as flat two-dimensional images. Yet in order to do so in a meaningful manner, so that the diagnosis will be accurate, it is essential that the geometric dilatation and distortion, in terms of change of angles and lengths, caused by this representation, will be minimal. However, since most surfaces of medical interest, such as colon, cortex, etc., are not isometric to the plane, a zero-distortion solution is seldom feasible. A reasonable solution to this problem is given by conformal maps ([8], [9]). Mapping the surface conformally to the (complex) plane preserves angles and therefore the local shape.

Naturally, the problem of conformal flattening of surfaces, in particular for medical imaging, has focused the interest of many researchers in the recent years, and there exists a vast literature covering the said problem (see Section 1.1 below). In fact, in all previous works, only approximations of conformal mappings have been achieved, and as such they all suffer from the presence of some amount of distorsion/dilatation. Therefore, one should aim to control the amount of inherent distortion. This can be achieved by quasi-isometric/quasi-conformal maps 
(i.e. maps that are almost isometries/conformal; precise definition will follow in Section 2). Practically, there is a tradeoff between the cost of an implementation on one hand and accuracy on the other. Common to all solutions is the fact, which cannot be avoided because of the inevitable distortion, that the more locally one is willing to focus, the more accurate the results become.

\section{$1.1 \quad$ Related Works}

As stated above, the problem of minimal distortion flattening of surfaces attracted, in recent years, a great attention and interest, due to its wide range of applications.

In this section we briefly review some of the methods that were proposed for dealing with this problem.

Variational Methods. Haker et al. ([8], [9]) introduced the use of a variational method for conformal flattening of CT/MRI 3-D scans of the brain/colon for the purpose of medical imaging. The method is essentially based on solving Dirichlet problem for the Laplace-Beltrami operator $\triangle u=0$ on a given surface $\Sigma$, with certain boundary conditions on $\partial \Sigma$. A solution to this problem is a harmonic (thus conformal) map from the surface to the (complex) plane. The solution suggested in [8] and [9] is a PL (piecewise linear) approximation of the smooth solution, achieved by solving a proper system of linear equations.

Circle Packing. Hurdal et al. ([10]) attempt to obtain such a conformal map by using circle packing. This relies on the ability to approximate conformal structure on surfaces by circle packings. The authors use this method for MRI brain images and conformally map them to the three possible models of geometry in dimension 2 (i.e. the 2-sphere, the Euclidian plane and the Hyperbolic plane). Yet, the method is applicable for surfaces which are topologically equivalent to a disk whereas the brain cortex surface is not. This means that there is a point of the brain (actually a neighborhood of a point), which will not map conformally to the plane, and in this neighborhood the dilatation will be infinitely large. Hurdal et al. solve this problem by removing the corpus callosum, thus obtaining a surface homeomorphic to a 1-punctured sphere, and thus conformally equivalent to a disk $([10],[11])$. An additional problem arises due to the necessary assumption that the surface triangulation is homogeneous in the sense that all triangles are equilateral. Such triangulations are seldom attainable.

Holomorphic 1-forms. Gu et al. ([6], [7], [5]) are using holomorphic 1-forms in order to compute global conformal structure of a smooth surface of arbitrary genus given as a triangulated mesh. holomorphic 1-forms are differential forms (differential operators) on smooth manifolds, which among other things can depict conformal structures. This method indeed yields a global conformal structure hence, a conformal parameterization for the surface however, computing homology basis is extremely time consuming. 
Angle Methods. In [12] Sheffer et al. parameterize surfaces via an angle based method in a way that minimizes angle distortion while flattening. However, the surfaces are assumed to be approximated by cone surfaces, i.e. surfaces that are composed from cone-like neighborhoods.

To summarize, all the methods described above compute only approximation to conformal mappings, therefore producing only quasi-conformal mappings, with no precise estimates on the dilatation.

In this paper we propose yet another solution to this problem. The proposed method relies on theoretical results obtained by Gehring and Väisalä in the 1960's ([4]). They were studying the existence of quasi-conformal maps between Riemannian manifolds. The basic advantages of this method resides in its simplicity, in setting, implementation and its speed. Additional advantage is that it is possible guarantee not to have distortion above a predetermined bound, which can be as small as desired, with respect to the amount of localization one is willing to pay (and, in the case of triangulated surfaces, to the quality of the given mesh). In fact, the proposed method is - to the best of our knowledge - the only algorithm capable of computing both length distortion and angle dilatation. The suggested algorithm is best suited to cases where the surface is complex (high and non-constant curvature) such as brain cortex/colon wrapping, or of large genus, such as skeleta, proteins, etc. Moreover, since together with the angular dilatation, both length and area distortions are readily computable, the algorithm is ideally suited for applications in Oncology, where such measurements are highly relevant.

The paper is organized as follows: In the next section we introduce the theoretical background, regarding the fundamental work of Gehring and Väisalä. Afterwards we describe our algorithm for surface flattening, based on their ideas. In Section 4 we present some experimental results of this scheme and in Section 5 we discuss possible extensions of this study.

\section{Theoretical Background}

\subsection{Basic Definitions}

Definition 1. Let $D \subset \mathbb{R}^{3}$ be a domain. A homeomorphism $f: D \rightarrow \mathbb{R}^{3}$ is called a quasi-isometry (or a bi-lipschitz mapping), if there exists $1 \leq C<\infty$, such that

$$
\begin{aligned}
& \frac{1}{C}\left|p_{1}-p_{2}\right| \leq\left|f\left(p_{1}\right)-f\left(p_{2}\right)\right|<C\left|p_{1}-p_{2}\right|, \text { for all } p_{1}, p_{2} \in D . \\
& C(f)=\min \{C \mid f \text { is a quasi - isometry }\} \text { is called the minimal distortion of } \\
& f(\text { in } D) \text {. }
\end{aligned}
$$

Remark 1. If $f$ is a quasi-isometry then $K_{I}(f) \leq C(f)^{2}$ and $K_{O}(f) \leq C(f)^{2}$ where $K_{I}(f), K_{O}(f)$ represent the inner, respective outer dilatation of $f$ (see see v. It follows that any quasi-isometry is a quasi-conformal mapping (while 
- evidently - not every quasi-conformal mapping is a quasi-isometry). Quasiconformal is the same as quasi-isometry where distances are replaced by angles.

Definition 2. Let $S \subset \mathbb{R}^{3}$ be a connected set. $S$ is called admissible iff for any $p \in S$, there exists a quasi-isometry $i_{p}$ such that for any $\varepsilon>0$ there exists a neighbourhood $U_{p} \subset \mathbb{R}^{3}$ of $p$, such that $i_{p}: U_{p} \rightarrow \mathbb{R}^{3}$ and $i_{p}\left(S \cap U_{p}\right)=D_{p} \subset \mathbb{R}^{2}$, where $D_{p}$ is a domain and such that $C\left(i_{p}\right)$ satisfies:

(i) $\sup _{p \in S} C\left(i_{p}\right)<\infty$;

and

$$
\text { (ii) } \sup _{p \in S} C\left(i_{p}\right)<1+\varepsilon \text {. }
$$

\subsection{The Projection Map}

Let $S$ be a surface, $\overline{\boldsymbol{n}}$ be a fixed unitary vector, and $p \in S$. Let $V \simeq D^{2}$, $D^{2}=\left\{x \in \mathbb{R}^{2} \mid\|x\| \leq 1\right\}$ be a disk neighbourhood of $p$. Moreover, suppose that for any $q_{1}, q_{2} \in S$, the acute angle $\measuredangle\left(q_{1} q_{2}, \overline{\boldsymbol{n}}\right) \geq \alpha$ (see Figure 2 ). We refer to the last condition as the Geometric Condition or Gehring Condition.

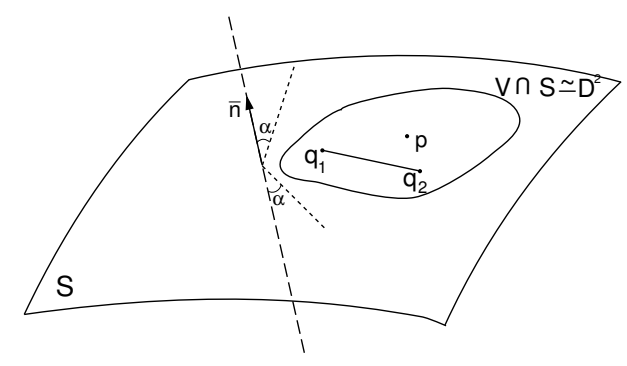

Fig. 1. The Geometric Condition

Then for any $x \in V$ there is a unique representation of the following form:

$$
x=q_{x}+u \overline{\boldsymbol{n}} ;
$$

where $q_{x}$ lies on the plane through $p$ which is orthogonal to $\overline{\boldsymbol{n}}$ and $u \in \mathbb{R}$.

Define:

$$
\operatorname{Pr}(x)=q_{x} .
$$

Note: $\overline{\boldsymbol{n}}$ need not be the normal vector to $S$ at $p$. 
By [4], Section 4.3 and Lemma 5.1, we have that for any $p_{1}, p_{2} \in S$ and any $a \in \mathbb{R}_{+}$the following inequalities hold:

$$
\frac{a}{A}\left|p_{1}-p_{2}\right| \leq\left|\operatorname{Pr}\left(p_{1}\right)-\operatorname{Pr}\left(p_{2}\right)\right| \leq A\left|p_{1}-p_{2}\right| ;
$$

where

$$
A=\frac{1}{2}\left[(a \csc \alpha)^{2}+2 a+1\right]^{2}+\frac{1}{2}\left[(a \csc \alpha)^{2}-2 a+1\right]^{2} .
$$

In particular for $a=1$ we get that

$$
C(f) \leq \cot \alpha+1
$$

and

$$
K(f) \leq\left(\left(\frac{1}{2}(\cot \alpha)^{2}+4\right)^{\frac{1}{2}}+\frac{1}{2} \cot \alpha\right)^{\frac{3}{2}} \leq(\cot \alpha+1)^{\frac{3}{2}}
$$

where

$$
K(f)=\max \left(K_{O}(f), K_{I}(f)\right)
$$

is the maximal dilatation of $f$.

The Geometric Condition From the discussion above we conclude that $S \subset$ $\mathbb{R}^{3}$ is an admissible surface if for any $p \in S$ there exists $\overline{\boldsymbol{n}}_{p}$ such that for any $\varepsilon>0$, there exists $U_{p} \simeq D^{2}$, such that for any $q_{1}, q_{2} \in U_{p}$ the acute angle $\measuredangle\left(q_{1} q_{2}, \overline{\boldsymbol{n}}_{p}\right) \geq \alpha$, where

$$
\text { (i) } \inf _{p \in S} \alpha_{p}>0
$$

and

$$
\text { (ii) } \inf _{p \in S} \alpha_{p}<\frac{\pi}{2}-\varepsilon
$$

Example 1. Any surface in $S \in \mathbb{R}^{3}$ that admits a well-defined continuous turning tangent plane at any point $p \in S$ is admissible.

\section{The Algorithm}

We will present in this section the algorithm that is used for obtaining a quasiisometric (flat) representation of a given surface. First assume the surface is equipped with some triangulation $T$. Let $N_{p}$ stand for the normal vector to the surface at a point $p$ on the surface.

Second, a triangle $\Delta$, of the triangulation must be chosen. We will project a patch of the surface quasi-isometrically onto the plane included in $\Delta$. This patch will be called the patch of $\Delta$, and it will consists of at least one triangle, $\Delta$ itself. 
There are two possibilities to chose $\Delta$, one is in a random manner and the other is based on curvature considerations. We will refer to both ways later. For the moment assume $\Delta$ was somehow chosen. After $\Delta$ is (trivially) projected onto itself we move to its neighbors. Suppose $\Delta^{\prime}$ is a neighbor of $\Delta$ having edges $e_{1}$, $e_{2}, e_{3}$, where $e_{1}$ is the edge common to both $\Delta$ and $\Delta^{\prime}$.

We will call $\Delta^{\prime}$ Gehring compatible w.r.t $\Delta$, if the maximal angle between $e_{2}$ or $e_{3}$ and $N_{\Delta}$ (the normal vector to $\Delta$ ), is greater then a predefined measure suited to the desired predefined maximal allowed distortion, i.e. $\max \left\{\varphi_{1}, \varphi_{2}\right\} \geq$ $\alpha$, where $\varphi_{1}=\measuredangle\left(e_{2}, N_{\Delta}\right), \varphi_{2}=\measuredangle\left(e_{3}, N_{\Delta}\right)$; (cf. (1), (2)).

We will project $\Delta^{\prime}$ orthogonally onto the plane included in $\Delta$ and insert it to the patch of $\Delta$, iff it is Gehring compatible w.r.t $\Delta$.

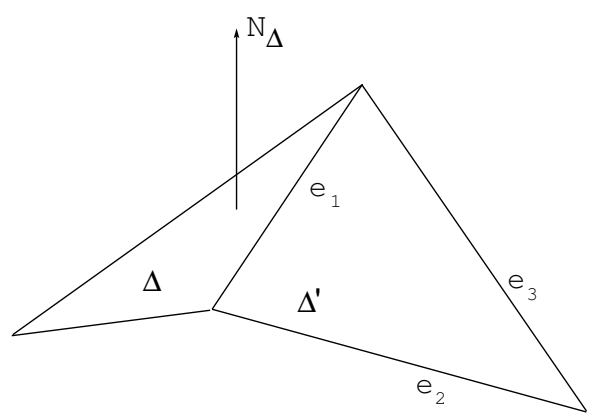

Fig. 2. Gehring Compatible Triangles

We keep adding triangles to the patch of $\Delta$ moving from an added triangle to its neighbors (of course) while avoiding repetitions, till no triangles can be added. If by this time all triangles where added to the patch we have completed constructing the mapping. Otherwise, chose a new triangle that has not been projected yet, to be the starting triangle of a new patch. A pseudocode for this procedure can be easily written.

Remark 2. There are two ways for choosing a base triangle for each patch. One is by curvature considerations, i.e. taking a triangle which the sum of the (magnitude of) curvatures of its vertices is minimal, and the other one is by randomly choosing a triangle for each new patch.

Remark 3. One should keep in mind that the above given algorithm, as for any other flattening method, is local. Indeed, in a sense the (proposed) algorithm gives a measure of "globality" of this intrinsically local process.

Remark 4. Our algorithm is best suited for highly folded surfaces, because of its intrinsic locality, on the one hand, and computational simplicity, on the other. 
However, on "quasi-developable" surfaces (i.e. surfaces that are almost cylindrical or conical) the algorithm behaves similar to other algorithms, with practically identical results.

\section{Experimental Results}

We now proceed to present some experimental results obtained by applying the proposed algorithm, both on synthetic surfaces and on data obtained from actual CT scans.

In each of the examples both the input surface and a flattened representation of some patch are shown. Details about mesh resolution as well as flattening distortion are also provided. The number of patches needed in order to flatten the surface is also given. In all images, the small rectangle shown on the surface represents a base triangle for the flattened patch. The colored area in each of the images represents the patch being flattened.

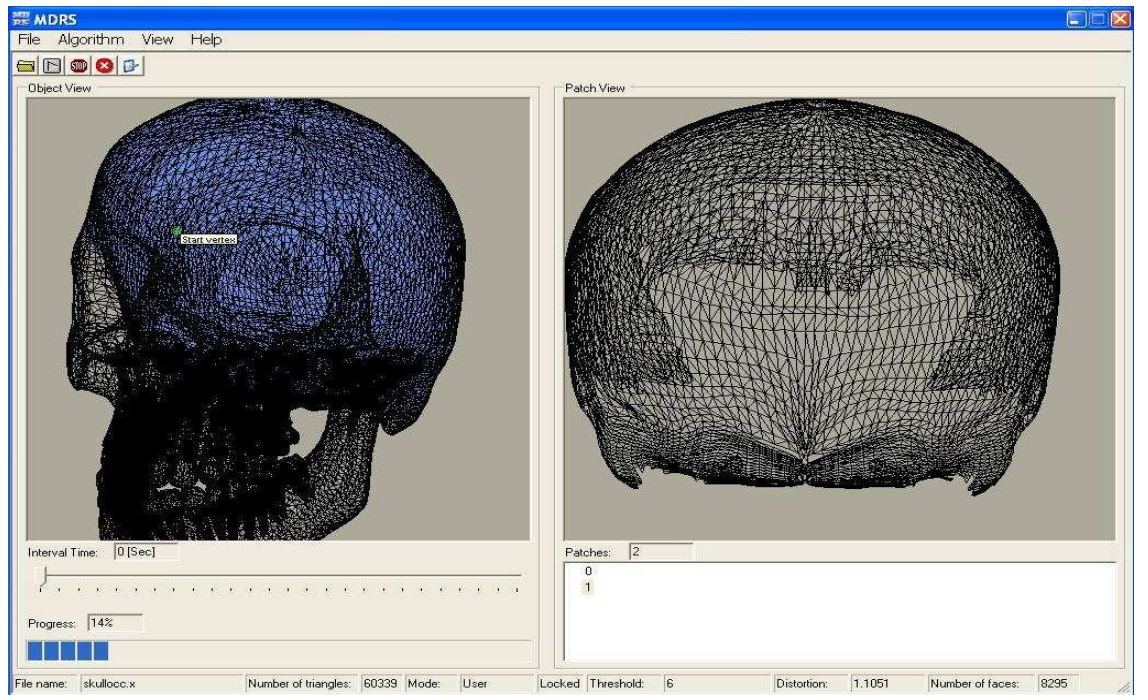

Fig. 3. Skull Flattening: The role of almost flat regions is accentuated. The resolution is of 60,339 triangles. Here $\alpha$ is $10^{\circ}$ and the dilatation is 1.1763 .

The algorithm was implemented in two versions, or more precisely two possible ways of processing, automatic versus user defined.

- Automatic means that the triangles serving as base points for the patches to be flattened are chosen automatically according to curvature, as stated in Remark 3.2. The discrete curvature measure employed is that of angular defect, due to its simplicity and high reliability (see [13]). 


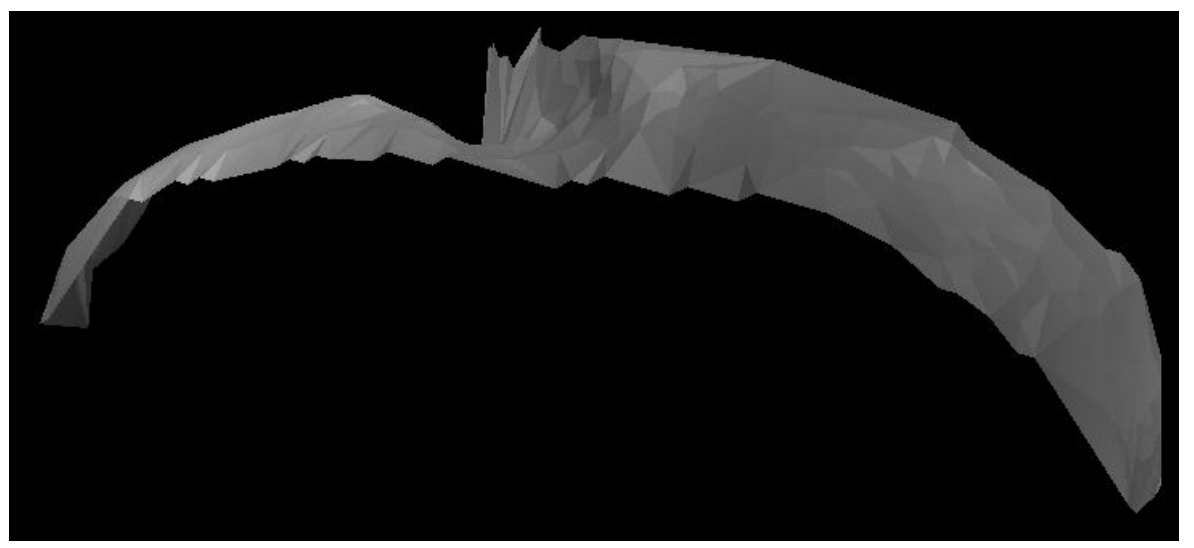

Fig. 4. Colon Section: Observe the highly folded region that a large number of patches while preserving a small dilatation. The image shows the back-side of the colon, whose flattened image is depicted in Fig. 5. (b). CT-data is in curtesy of Dr. Doron Fisher from Rambam Madical Center in Haifa.

- User defined means that at each stage the user chooses a base triangle for some new patch.

Since at this stage we did not address the problem of properly gluing of patches, in the following example of colon flattening, one can see the appearance of holes in the flattened presentation caused by artificially gluing neighboring patches to each other. We refer to this problem in the next section.

Experiments have shown that results of the automatic process are similar, in terms of the dilatation, to those obtained from the user defined process yet, in order to flatten entire surface in the user defined method one needs in average $25 \%$ more patches.

\section{Concluding Remarks and Future Study}

Sampling and flattening of folded surfaces embedded in higher dimensional Riemannian manifolds combines several important facets and problems encountered in image processing and analysis of surfaces. In our broader study [2], we deal with the issues of nonuniform smoothing and sampling. Here we assumed that a proper sampling and triangulation of the surfaces are given. the emphasis was therefore on quasi-conformal and quasi-isometric aspects of the mapping between Riemannian manifolds. While the theory is general and applicable to mapping from any higher to lower dimensional manifolds, here we presented a specific algorithm developed for the case of mapping from a three-dimensional to two-dimensional flat surface.

From the implementation results it is evident that this algorithm while being simple to program as well as efficient, also gives good flattening results and main- 


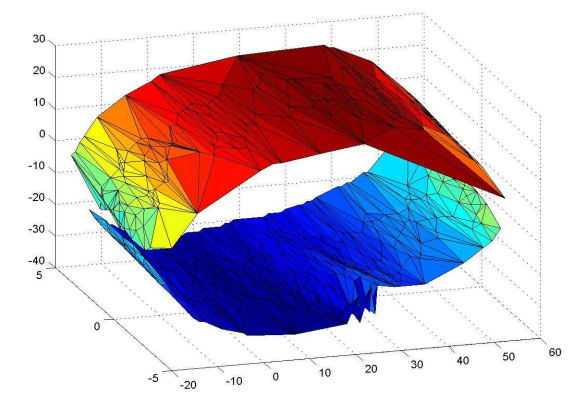

(a)

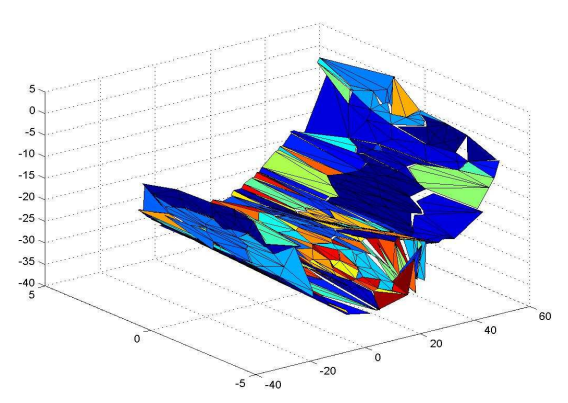

(b)

Fig. 5. Colon CT-Images: (a) Triangulated colon surface taken from 3 slices of human colon scan and (b) One half of the colon, after flattening. One is able to observe the holes caused by improper gluing of neighbouring patches.

tains small dilatations even in areas where curvature is large and good flattening is a challenging task. Moreover, since there is a simple way to assess the resulting dilatation/distorsion, the algorithm was implemented in such a way that the user can set in advance an upper bound on the resulting dilatation/distorsion. Let us stress once more that, to the best of our knowledge, our method represents the only algorithm capable of computing both length distortion and angle dilatation.

An additional advantage of the presented algorithm is related to the fact that, contrary to some of the related studies, no use of derivatives is made. Consequently, the algorithm does not suffer from typical drawbacks of derivative computations like lack of robustness, etc.

Moreover, since no derivatives are employed, no smoothness assumption about the surface to be flattened are made, which makes the algorithm presented herein ideal for use in cases where smoothness is questionable (to say the least).

The algorithm may be practical for applications where local yet, good analysis is required such as medical imaging with the emphasis on flattened representation of the brain and the colon (virtual colonoscopy) - see [1]. Further study is currently undertaken.

The main issue for further investigation, remains the transition from local to global in a more precise fashion, i.e. how can one glue two neighbouring patches while keeping fixed bounded dilatation. In more technical terms, this amounts to actually computing the holonomy map of the surface - see [14]. Computing holonomy tells you exactly how to match-up two areas of a surface having different conformal characteristics so that a bigger patch with controlled quasi-conformal behaviour will be obtained. This is also under current investigation.

Evidently, as can be seen in Fig. 5, of the colon flattening example, one can have two neighbouring patches, with markedly different dilataions/distorsions, 
which results in different lengths for the common boundary edges. Therefore, "cuts" and "holes" appear when applying a "naive" glueing.

We conclude by remarking that while the the application presented here is for $2 D$-images of $3 D$-surfaces, the results of Gehring and Väisalä are stated and proven for any dimension (and co-dimension). Therefore, implementations for higher dimensions are also in progress.

\section{Acknowledgment}

Emil Saucan is supported by the Viterbi Postdoctoral Fellowship. Research is partly supported by the Ollendorf Minerva Center.

The authors would like to thank Ofir Zeitoun and Efrat Barak and Amiad Segal and Ronen Lev for their dedicated and skillful programming of the algorithms.

\section{References}

[1] Appleboim, E., Saucan E., and Zeevi, Y. Minimal-Distortion Mappings of Surfaces for Medical Imaging, Proceedings of VISAPP 2006, to appear.

[2] Appleboim, E., Saucan, E., and Zeevi, Y.Y. On Sampling and Reconstruction of Surfaces, Technion CCIT Report, 2006.

[3] Caraman, P. n-Dimensional Quasiconformal (QCf) Mappings, Editura Academiei Române, Bucharest, Abacus Press, Tunbridge Wells Haessner Publishing, Inc., Newfoundland, New Jersey, 1974.

[4] Gehring, W. F. and Väisälä, J. The coefficients of quasiconformality, Acta Math. 114, pp. 1-70, 1965.

[5] Gu, X. Wang, Y. and Yau, S. T. Computing Conformal Invariants: Period Matrices, Communications In Information and Systems, Vol. 2, No. 2, pp. 121-146, December 2003.

[6] Gu, X. and Yau, S. T. Computing Conformal Structure of Surfaces, Communications In Information and Systems, Vol. 2, No. 2, pp. 121-146, December 2002.

[7] Gu, X. and Yau, S. T. Global Conformal Surface Parameterization, Eurographics Symposium on Geometry Processing, 2003.

[8] Haker, S. Angenet, S. Tannenbaum, A. Kikinis, R. Non Distorting Flattening Maps and the 3-D visualization of Colon CT Images, IEEE Transauctions on Medical Imaging, Vol. 19, NO. 7, July 2000.

[9] Haker, S. Angenet, S. Tannenbaum, A. Kikinis, R. Sapiro, G. Halle, M. Conformal Surface Parametrization for Texture Mapping, IEEE Transauctions on Visualization and Computer Graphics, Vol. 6, NO. 2, June 2000.

[10] Hurdal, M. K., Bowers, P. L., Stephenson, K., Sumners, D. W. L., Rehm, K., Schaper. K., Rottenberg, D. A. Quasi Conformally Flat Mapping the Human Crebellum, Medical Image Computing and Computer-Assisted Intervention -MICCAI'99, (C. Taylor and A. Colchester. eds), vol. 1679, Springer-Verlag, Berlin, 279-286, 1999.

[11] Stephenson, K. personal communication.

[12] Sheffer, A. de Stuler, E. Parametrization of Faceted Surfaces for Meshing Using Angle Based Flattening, Enginneering with Computers, vol. 17, pp. 326-337, 2001. 
[13] Surazhsky, T., Magid, E., Soldea, O., Elber, G. and Rivlin E. A Comparison of Gaussian and Mean Curvatures Estimation Methods on Triangular Meshes, Proceedings of the IEEE International Conference on Robotics and Automation. Taipei, Taiwan, pp 1021-1026, September 2003.

[14] Thurston, W. Three-Dimensional Geometry and Topology, vol.1, (Edited by S. Levy), Princeton University Press, Princeton, N.J. 1997.

[15] Väisalä, J. Lectures on n-dimensional quasiconformal mappings, Lecture Notes in Mathematics 229, Springer-Verlag, Berlin - Heidelberg - New-York, 1971. 

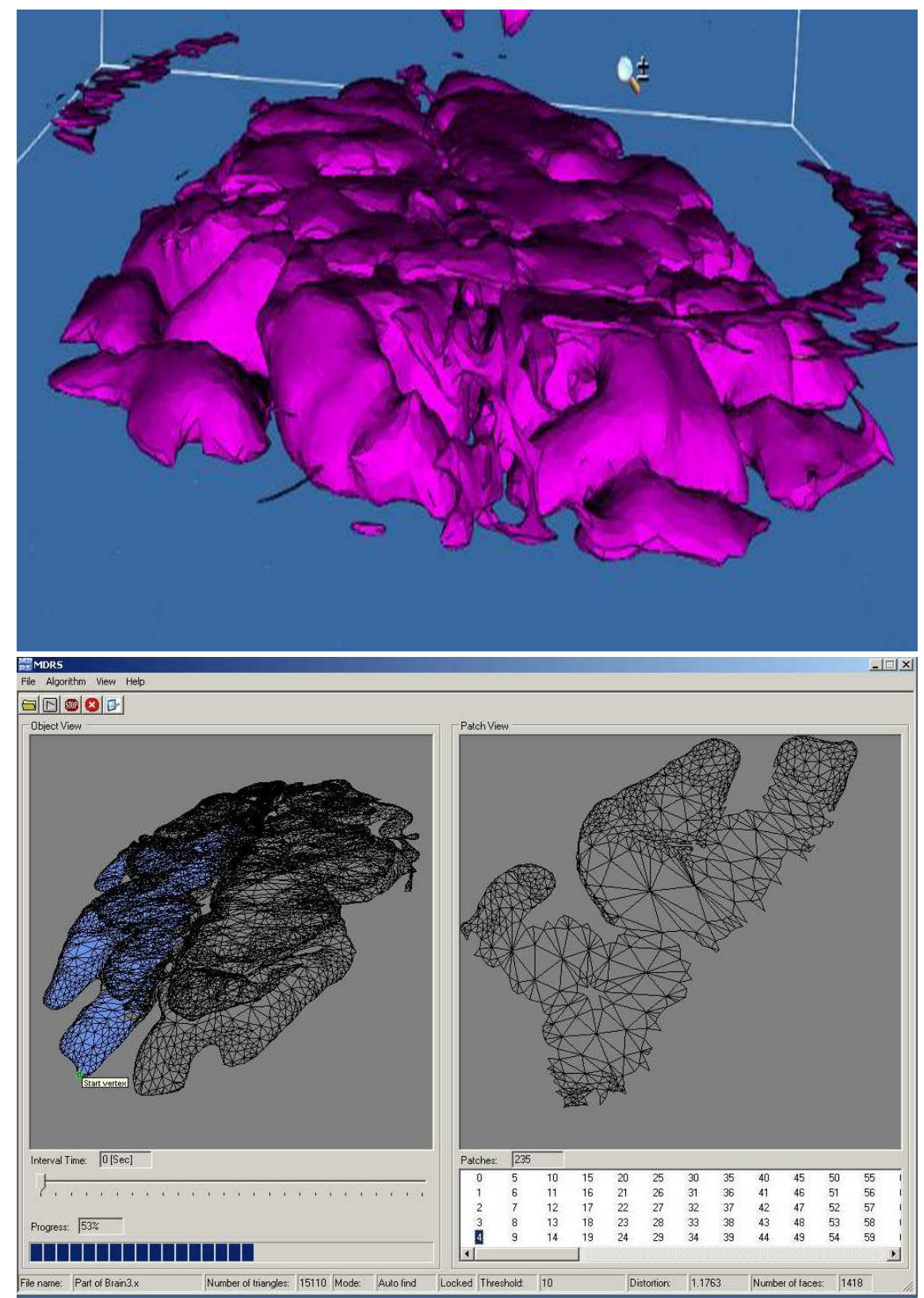

Fig. 6. Cerebral Cortex Flattening: A patch obtained in the flattening of the parietal region. The resolution is 15.110 triangles, the angle chosen is $5^{\circ}$, producing a dilatation of 1.0875 . 